ERRATUM

\title{
Structural plasticity and reorganisation in chronic pain
}

Rohini Kuner and Herta Flor

Nature Reviews Neuroscience 18, 20-30 (2017)

In Box 1 and on page 26 of this article, in the section Activity-dependent 'switches', $A \beta$ afferents were incorrectly defined.

This has been corrected in the online version of the article. We apologize to the authors and readers for this error. 